\title{
A quantitative investigation of gonadal feminization by diethylstilboestrol of genetically male embryos of the quail Coturnix coturnix japonica
}

\author{
F. M. R. Perrin, S. Stacey, A. M. C. Burgess and U. Mittwoch* \\ Department of Anatomy, Queen Mary and Westfield College, Mile End Road, London, E1 4NS, UK
}

\begin{abstract}
The effect of diethylstilboestrol on gonad development in quail embryos has been quantitatively analysed. Quail embryos at 4 days of incubation were treated with diethylstilboestrol (DES), using the egg dipping method. At 10 days of incubation, embryos were removed and killed by decapitation. Tissues were prepared for chromosome analysis, and the parts of the abdomen containing the gonads were prepared for serial sectioning and quantitative assessment. Left gonads of DES-treated male embryos resembled ovaries histologically, while their right gonads were markedly reduced in size. Right gonads of DES-treated female embryos were also further reduced by treatment with DES. There was no statistically significant effect by DES treatment on the size of left gonads, although the ratio of left compared with right gonadal volumes was highly significant. Since, in birds, the left embryonic gonad has ambisexual potential, while the potential of the right gonad is exclusively masculine, these results exemplify the adverse effect exerted by oestrogen on male sexual development in vertebrates.
\end{abstract}

\section{Introduction}

Studies on the genetics of sex differentiation in birds lag behind those of mammals and of reptiles. Yet the avian embryo offers advantages in both accessibility and availability and is thus a potentially useful candidate for studying the effects of exogenous substances on the development of the gonads.

A characteristic feature of sexual development in birds is the asymmetry of their gonads. In most species, ovaries are confined to the left side, while the right gonad of females remains rudimentary, but, in certain circumstances, has the potential to develop into a testicular structure (Lillie, 1952). Moreover, in the early embryo, the left gonad in both sexes has the potential to develop ovarian tissue, while the only potential of the right gonad is for testicular development. Evidently, the chromosomal sex-determining mechanism is more open to environmental modification than is that of mammals.

Wolff and Ginlinger (1935) reported that injecting diethylstilboestrol (DES) into incubating chick eggs produces a feminization of male chicks. A simplified method is described by Seltzer (1956; cited by Pincus, 1958), which, in place of injection, substitutes dipping the eggs into the solutions to be tested. Dipping quail (Coturnix coturnix) eggs that have been incubated for 4 days into an alcoholic solution of $40 \mathrm{mg}$ per $100 \mathrm{ml}$ ethanolic DES achieves a modification of male gonads (Haffen, 1965). Instead of appearing symmetrical, the left gonad of treated embryos assumes the appearance of an ovary, while the right gonad is reduced. The feminization of quail embryos

\footnotetext{
*Correspondence.

Received 30 June 1994.
}

appears to be more pronounced than in chicks; whereas feminized chicks revert to male sex characteristics soon after hatching (Wolff and Haffen, 1961), sex reversal in quails seems more stable. Haffen (1965) reports that an 8-week-old male had an ovotestis containing follicles.

Here, we report a detailed quantitative investigation of DES-treated and control gonads in 10-day-old quail embryos, the genetic sex of which has been verified by chromosome analysis. As in other birds, the sex chromosomes of males consist of two equal $\mathrm{Z}$ chromosomes, while females have one $\mathrm{Z}$ chromosome and a smaller $\mathrm{W}$ chromosome.

\section{Materials and Methods}

Fertilized quail eggs were obtained from a breeding colony maintained by J. Bee at the Royal Veterinary College, London. Each batch of eggs was divided into three groups and placed in randomized positions in an incubator kept at $37.5^{\circ} \mathrm{C}$. After 4 days of incubation, one group of eggs was dipped into a solution of $40 \mathrm{mg}$ DES in $100 \mathrm{ml}$ of $95 \%$ (v/v) ethanol, while the second group was dipped into $95 \%$ ethanol, and the third group was left untreated. Dipping was carried out by placing each egg, with its pointed end downwards, into a net and immersing it along two-thirds of its length for $10 \mathrm{~s}$. The eggs were then returned to the incubator and left for another 6 days. At the end of this period, the embryos were removed, and their crown-rump lengths were measured, using digital calipers. The heads of the embryos were set up for chromosome analysis, using the method of Tuinen and Valentine (1983), while the parts of the bodies containing the gonads were prepared 

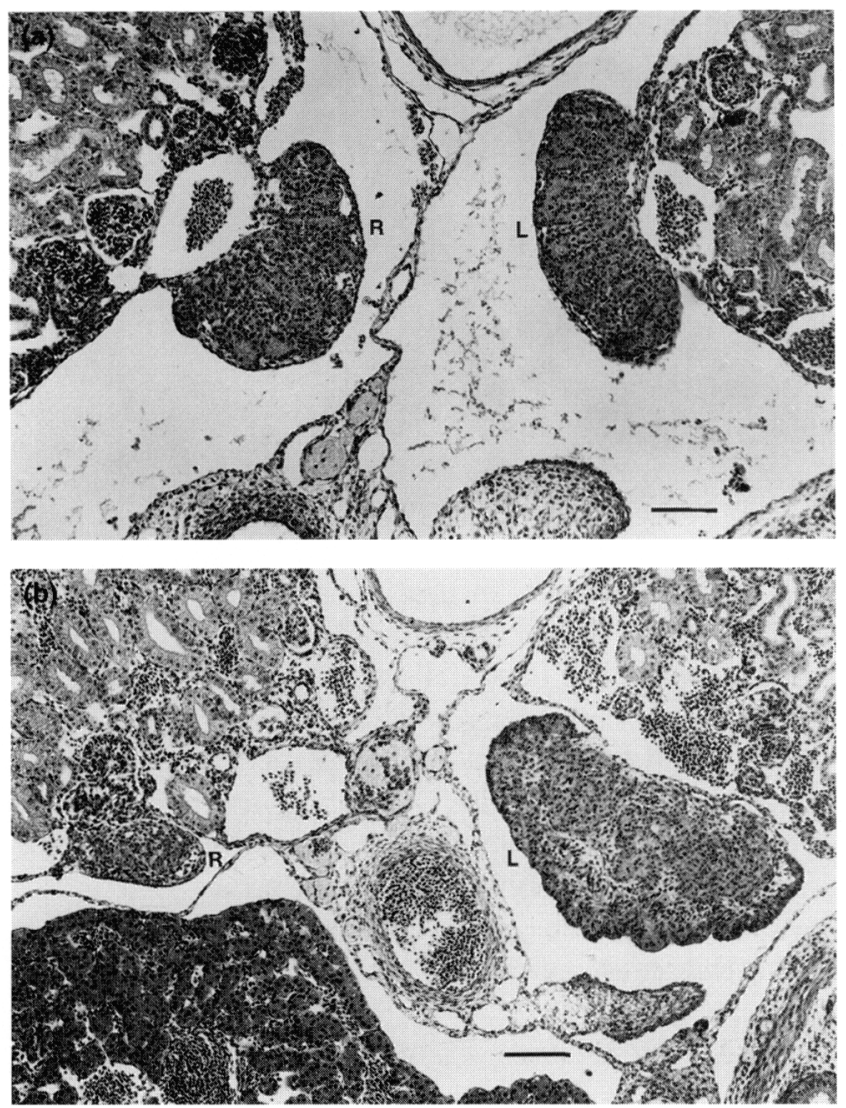

Fig. 1. Transverse sections of male quail embryos in the region of the gonads. (a) Ethanol-treated control embryo, (b) diethylstilboestrol (DES)-treated embryo. L: left gonad; R: right gonad. Scale bar represents $80 \mu \mathrm{m}$.

for serial transverse sectioning at $7 \mu \mathrm{m}$ and staining in haematoxylin and eosin.

Volumes of left and right gonads were computed from section areas as described by Baker et al. (1993), using a Leitz camera lucida and a digitizing tablet connected to an Opus computer. Areas of between 30 and 40 sections spanning the entire gonad were measured and the sum of the areas was converted into volumes by multiplying by the effective section thickness (for example, if every eighth section was measured, the effective section area would be $56 \mu \mathrm{m}$ ); this method is unaffected by the angle of sectioning. The results obtained for DES-treated gonads and the two types of controls were compared by Student's $t$ test.

\section{Results}

Mean crown-rump lengths varied between 18.1 and $19.8 \mathrm{~mm}$ in different groups but the differences were not significant; these data are not shown.

\section{Male embryos}

The histological appearance of DES-treated and control gonads in genetically male embryos is illustrated in Fig. I.
Whereas in untreated and ethanol-treated embryos, left and right gonads appeared to be symmetrical (Fig. 1a), after DES treatment, the right gonad was markedly reduced in size, while the comparatively much larger left gonad assumed the general appearance of an ovary (Fig. Ib). It had a more rugose outline than did the control gonads, and signs of an incipient ovarian cortex could be made out along its lower edge.

The difference in size between left and right DES-treated gonads was highly significant, whereas there were no significant differences between left and right untreated or ethanol-treated gonads (Table 1). Neither treatment exerted a significant effect on the size of the left gonad, and there was no significant difference in size between untreated and ethanoltreated right gonads. By contrast, treatment with DES caused a highly significant reduction in the size of the right gonad.

\section{Female embryos}

All genetically female embryos had a left ovary and a reduced right gonad, and there was no obvious effect produced by any of the treatments (Table 2). The difference in size between left and right gonads is highly significant in all groups, and the effect of DES in further reducing the size of the right gonad is also significant.

\section{Comparison between male and female embryos}

Figure 2 illustrates the difference in gonadal volume obtained when the mean volumes of female gonads are subtracted from those of male gonads in any one group. In untreated and ethanol-treated embryos, the results are negative for left gonads and positive for right gonads, but this effect is not evident in DES-treated embryos. In control embryos, females have a larger left gonad and a smaller right gonad than do males. This relationship is no longer seen in embryos treated with DES, in which neither difference is significantly different from zero.

\section{Discussion}

Our results confirm the reports by Haffen (1965) and Scheib et al. (1981) that the dipping of quail eggs into an alcoholic solution of DES results in the partial feminization of embryos and hatched birds. We have shown that 10-day-old male embryos exposed to DES show a marked reduction in the size of the right gonad, and that female embryos show a somewhat smaller, but still significant, reduction in the size of the right gonad. By contrast, the hormone did not reduce the size of the left gonad, the morphology of which was similar in some ways to an ovary. This gonad was a little larger than in the controls, but the difference was not significant. In female embryos treated with DES, volumes of both gonads were reduced, but the difference was significant only for the right gonad. The DES-induced changes in the growth patterns of the four types of gonad abolished the normal relationship seen in the controls, in which the left ovary is larger than the testis, and the right rudimentary gonad of the female is very much smaller than a testis. 
Table 1. Volumes of left and right gonads in 10-day-old genetically male embryos of Coturnix coturnix japonica treated with $40 \mathrm{mg}^{\mathrm{DES} \mathrm{ml}} \mathrm{m}^{-1}$, with vehicle, and in untreated controls

\begin{tabular}{lcccc}
\hline Treatment & $n$ & $\begin{array}{c}\text { Gonadal volumes }\left(\mathrm{mm}^{3}\right) \pm \text { SEM } \\
\text { Left }\end{array}$ & $\begin{array}{c}\text { Significance } \\
\text { Right }\end{array}$ \\
\hline Untreated & 5 & $0.1014 \pm 0.0053$ & $0.1019 \pm 0.0080$ & n.s. \\
Ethanol & 5 & $0.0883 \pm 0.0144$ & $0.0762 \pm 0.0116$ & n.s. \\
DES & 7 & $0.1228 \pm 0.0149$ & $0.0310 \pm 0.0069$ & $* * *$ \\
$\begin{array}{l}\text { Significance } \\
\text { Untreated versus Ethanol }\end{array}$ & & n.s. & n.s. & $* * *$ \\
$\quad$ Untreated versus DES & n.s. & $* *$ & \\
$\quad$ Ethanol versus DES & n.s. & & \\
& & &
\end{tabular}

DES: diethylstilboestrol.

$*^{* *} 0.001<P<0.01,{ }^{* * *} P<0.001$.

Table 2. Volumes of left and right gonads in 10-day-old genetically female embryos of Coturnix coturnix japonica treated with $40 \mathrm{mg} \mathrm{DES} \mathrm{ml}^{-1}$, with vehicle, and in untreated controls

\begin{tabular}{lcccc}
\hline Treatment & $n$ & \multicolumn{2}{c}{$\begin{array}{c}\text { Gonadal volumes }\left(\mathrm{mm}^{3}\right) \\
\text { Left }\end{array}$} & $\begin{array}{c}\text { Significance } \\
\text { Right } \\
\text { Left versus right }\end{array}$ \\
\hline Untreated & 9 & $0.1632 \pm 0.0254$ & $0.0294 \pm 0.0082$ & $* * *$ \\
Ethanol & 4 & $0.138 \pm 0.0134$ & $0.0282 \pm 0.0086$ & $* * *$ \\
DES & 7 & $0.0933 \pm 0.0141$ & $0.0171 \pm 0.0056$ & $* * *$ \\
$\begin{array}{l}\text { Significance } \\
\quad \text { Untreated versus ethanol }\end{array}$ & & n.s. & n.s. & $*$ \\
$\quad$ Untreated versus DES & n.s. & $*$ & \\
$\quad$ Ethanol versus DES & & n.s. & & \\
\hline
\end{tabular}

DES: diethylstilboestrol.

$* 0.02<P<0.05, * * * P<0.001$.

The difference in gonadal volumes between untreated and ethanol-treated embryos was not significant in any of the four types of gonad. However, ethanol-treated gonads were reduced in size in all four groups, suggesting that the alcohol may have a slight inhibitory effect on gonadal development; this possibility needs to be tested with a larger number of samples. The only increase in gonadal volume, albeit statistically not significant, was found in the left gonads of DEStreated male embryos, which became feminized as a result of the treatment.

In young chick embryos it can be seen clearly in both sexes that the left gonad has an incipient ovarian cortex and is thus potentially hermaphrodite. The cortex normally regresses in male embryos and is altogether absent in the right gonad of either sex, the only potential of which is to develop into a testis (Mittwoch, 1973, 1986). The feminization by DES of the left gonad of male embryos illustrates its fundamentally hermaphroditic nature. The marked reduction of the right gonad of male embryos exemplifies the deleterious nature of oestrogen on the developing testis and, to a lesser extent, this is also seen in the reduction in size of the right female gonad, which has the potential to develop into a testis.

Treating pregnant rats and mice with oestrogen is known to give rise to cryptorchidism (Hadžiselimovič et al., 1980) and there is evidence in human males that the administration of oestrogen to mothers during pregnancy also results in an increased incidence of cryptorchidism and inguinal hernia (Depuie, 1984). A rise in exogenous oestrogen acting on the male fetus has been suggested as a contributory factor for a possible secular fall in the number of spermatozoa and rises in disorders of the testis and the male reproductive tract, including testicular cancer, hypospadias and cryptorchidism (Giwersman et al., 1993). Since oestrogens are normally present in the uterine environment during pregnancy, the fast development of the male embryo and the early secretion of testosterone by the fetal testis, before high oestrogen concentrations are established, may be a necessary adaptation in the development of eutherian males (Mittwoch, 1993).

Failure of male sex differentiation in the presence of oestrogen appears to be widespread among vertebrates. In the red-eared slider, Trachemys scripta, a reptile with temperaturedependent sex determination, Wibbels et al. (1993) reported that eggs incubated at the male-producing temperature of $26^{\circ} \mathrm{C}$ and treated with oestradiol develop as phenotypic females. In another turtle, Caretta caretta, with a similar temperaturedependent system of sex determination, Harry and Williams (1991) reported different growth patterns for the male and female urogenital system during the sex-determining period.

Quantitative studies of the effect of oestrogen on the developing urogenital system of the quail offer considerable 


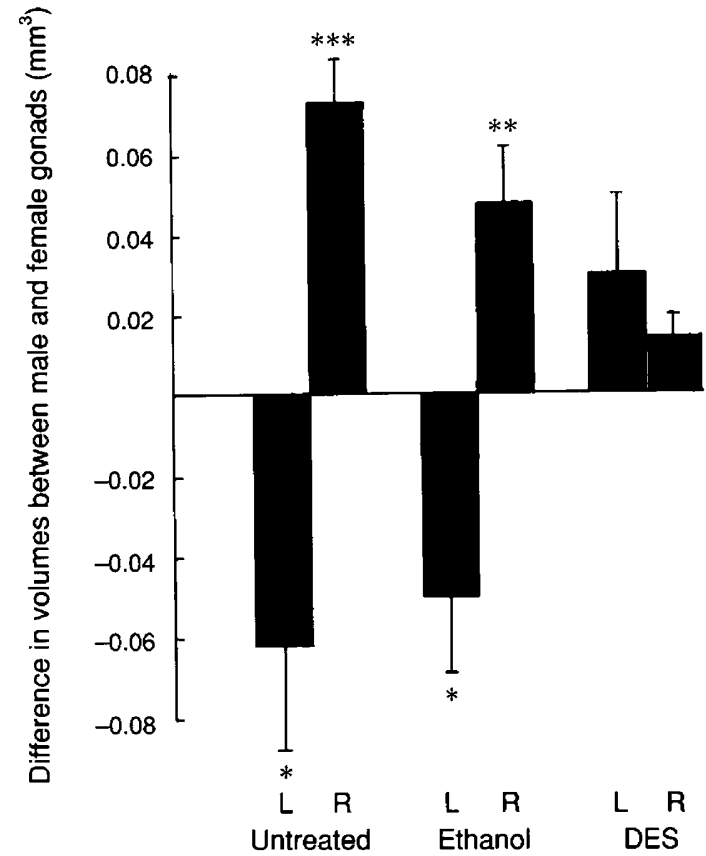

Fig. 2. Differences in size obtained after subtracting mean volumes of female gonads from those of male gonads in control and diethylstilboestrol (DES)-treated quail embryos (means + SEM). L: left gonad; $\mathrm{R}$ : right gonad. ${ }^{*} 0.02<P<0.05 ;{ }^{* *} 0.001<P<0.01 ;{ }^{* * *} P<0.001$.

potential for a better understanding of the different mechanisms operating in the process of sex determination in vertebrates (Mittwoch and Burgess, 1991). An important theoretical question that needs to be addressed is whether the asymmetric response to DES by the two gonads is caused by an initial difference in the presence of receptors, and whether the primary effect of oestrogen is the ovarian transformation of the left gonad or the inhibition of the right gonad.

The authors thank A. Aldrich and P. Hazell for preparing the photomicrographs, A. J. Lee for drawing the diagram and C. Turner for help with the manuscript. Financial support was provided by the Nuffield Foundation and the Leverhulme Trust.

\section{References}

Baker PJ, Moore HDM, Burgess AMC and Mittwoch U (1993) Gonadal sex differentiation in embryos and neonates of the marsupial, Monodelphis domestica: arrest of testis development in post-term embryos Journal of Anatomy 182 267-273

Depuie RH (1984) Maternal and gestational factors affecting the risk of cryptorchidism and inguinal hernia International journal of Epidemiology 13 $311-318$

Giwersman A, Carlsen E, Keiding N and Skakkebæk NE (1993) Evidence of increasing incidence of abnormalities of the human testis: a review Environmental Health Supplements 101 (Supplement 2) 65-71

Hadžiselimovič F, Herzog B and Krušlin E (1980) Estrogen-induced cryptorchidism in animals Clinics in Andrology 3 166-174

Haffen K (1965) Intersexualité chez la caille (Coturnix coturnix). Obtention d'un cas ovulaire par un mâle génétique Comptes Rendus de l'Académie des Sciences de Paris $2613876-3879$

Harry JL and Williams KL (1991) Differential growth of male and female urogenital systems of Caretta caretta, within the sex-determining period Journal of Experimental Zoology 258 204-2II

Lillie FR (1952) Development of the Chick (3rd Edn) (Revised by HL Hamilton). Henry Holt, New York

Mittwoch U (1973) Genetics of Sex Differentiation pp 119-132. Academic Press, New York

Mittwoch U (1986) Males, females and hermaphrodites Annals of Human Genetics 50 103-121

Mittwoch U (1993) Blastocysts prepare for the race to be male Human Reproduction 8 1550-1555

Mittwoch U and Burgess AMC (1991) How do you get sex? Journal of Endocrinology 128 329-331

Pincus G and Hopkins T (1958) The effects of various estrogens and steroid substances on sex differentiation in the fowl Endocrinology 62 111-118

Scheib D, Guichard A, Mignot THM and Cedard L (1981) Steroidogenesis by gonads of normal and of diethylstilbestrol-treated embryos: radioimmunoassays on organ cultures General and Comparative Endocrinology 43 519-526

Tuinen $\mathrm{P}$ and Valentine $\mathrm{M}$ (1983) A non-invasive technique of avian tissue culture (feather pulp) for banded chromosome preparations Mammalian Chromosome Newsletter 23 182-184

Wibbels T, Gideon P, Bull JJ and Crews D (1993) Estrogen- and temperatureinduced medullary cord regression during gonadal differentiation in a turtle Differentiation 53 149-154

Wolff E and Ginlinger A (1935) Sur la transformation des poulets mâles en intersexués par injection d'hormone femelle (folliculine) aux embryons Archives d'Anatomie, Histologie et Embryologie 20 219-278

Wolff E and Haffen K (1961) Sur la féminisation induite par les gonades mâles intersexués Archives d'Anatomie, Histologie de Embryologie 44 (Supplément) 273-302 